\title{
Arthropods as potential vectors of African swine fever virus outbreaks in pig farms in the Republic of Korea
}

\author{
Hachung Yoon $^{1}$ (D) | Seong-Keun Hong ${ }^{1}$ | llseob Lee $^{1}$ | Deuk-Soo Choi ${ }^{2}$ | \\ Jong-Ho Lee ${ }^{3}$ | Eunesub Lee ${ }^{1}$ Sung-Hwan Wee ${ }^{4}$
}

\author{
${ }^{1}$ Veterinary Epidemiology Division, Animal \\ and Plant Quarantine Agency, Gimcheon, \\ Republic of Korea \\ ${ }^{2}$ Plant Quarantine Technology Center, \\ Animal and Plant Quarantine Agency, \\ Gimcheon, Republic of Korea \\ ${ }^{3}$ Plant Pest Control Division, Animal and \\ Plant Quarantine Agency, Gimcheon, \\ Republic of Korea \\ ${ }^{4}$ Department of Animal Disease Control and \\ Quarantine, Animal and Plant Quarantine \\ Agency, Gimcheon, Republic of Korea

\section{Correspondence} \\ Hachung Yoon, Animal and Plant Quarantine \\ Agency, Gimcheon, Gyeonsangbuk-do, \\ Republic of Korea. \\ Email: heleney@korea.kr \\ Funding information \\ This study was financially supported by \\ the Animal and Plant Quarantine Agency \\ of the Republic of Korea (R\&D Project No.: \\ I-1543068-2021-22-01).
}

\begin{abstract}
The seasonality of African swine fever (ASF) in the summers and outbreaks in farms with high biosecurity levels suggest that the ASF virus (ASFV) may be transmitted by arthropod vectors. Arthropods were collected in this study from 14 pig farms with ASF outbreaks in Korea in 2019 to explore the role of arthropods as potential ASFV vectors. A total of 28,729 arthropods, including 28,508 (99.2\%) Diptera, were collected using blacklight traps, insect nets and yellow sticky strips. All arthropod samples were negative for ASFV genomic DNA according to laboratory tests using real-time polymerase chain reaction. This result may reflect the effects of immediate control measures following the detection of farms with ASF outbreaks in the early phase of infection in Korea.
\end{abstract}

\section{KEYWORDS}

African swine fever, arthropod, pig farm, Republic of Korea, transmission

\section{1 | INTRODUCTION}

African swine fever (ASF) is a haemorrhagic fatal disease in both domestic pigs and wild boars. Recently, there has been a drastic increase in the areas affected by ASF in Asia, Europe and Africa. ASF outbreaks in the Republic of Korea (hereafter Korea) were confirmed in 16 pig farms: 14 farms in 2019 (September 16 to October 9) and 2 farms in 2020 (October 8 and October 9) (Kim et al., 2021; Yoon et al., 2020). The ASF virus (ASFV) in wild boar was first detected in a carcass found in the demilitarized zone on October 2, 2019 (Jo \& Gortazar, 2020). Moreover, reports on the detection of ASF cases in wild boars have been continued (Lee et al., 2021; Vergne et al., 2020). Both farms with ASF outbreaks and wild boar cases were located near the border of the Democratic People's Republic of Korea (North Korea) (Kim et al., 2020; Yoo et al., 2020).

The seasonality of ASF, with cases concentrated over the summer in Europe, combined with outbreaks occurring in farms with high levels of biosecurity suggests that ASFV may be transmitted by arthropod vectors (Petrasiunas et al., 2018). Arthropods exist worldwide, including Korea. In this study, arthropods were collected from pig farms with ASF outbreaks to explore their role as potential ASFV vectors. 


\section{2 | MATERIALS AND METHODS}

Arthropods were caught by performing 111 operations between September 27 and October 31, 2019 in 14 farms (12 commercial and 2 backyard farms) with ASF outbreaks confirmed in 2019. Arthropod specimens were collected using blacklight traps (UV-LED Blackholeplus ${ }^{\circledR}$, Biotrap Ltd.), insect nets and yellow sticky traps. Any specific arthropod was targeted. Traps and nets were installed inside the pig house (on the pole of the pigpen and the top of the feedstuff), on the outer wall of the pig house and at the composting site to collect faeces. These were left in place for at least $24 \mathrm{hr}$ before the trapped arthropods were retrieved.

Arthropods collected using blacklight traps and insect nets were classified in the field and confirmed in the laboratory using Optinity KS-200® (Seoul, Korea) with 0.8-5x magnification. Yellow sticky traps were directly sent to the laboratory without onsite classification. Arthropods were morphologically identified and classified (based on scientific name, species or genus; Table S1) using the indented key. Same types of arthropods caught in the same device on the same day in each farm were pooled. The number of samples within each pool was 9 in median (2 for 1st quartile 2, 36.5 for 3rd quartile). The pooled specimens were homogenized in a lysis buffer with a TissueLyser II ${ }^{\circledR}$ (Qiagen, Hilden) for the detection of ASFV. Viral DNA extraction was carried out using the Maxwell Viral Total Nucleic Acid Purification Kit ${ }^{\circledR}$ (Promega) and TaqMan ${ }^{\circledR}$ (Applied Biosystems) real-time polymerase chain reaction using a set of primers (forward primer: $5^{\prime}-\mathrm{CT}$ GCT-CATGG-TATCA-ATCTTATCGA-3'; reverse primer: 5'-GATAC-C ACAA-GATC(AG)-GCCGT-3'; and probe: 5'-[6-carboxy-fluorescein (FAM)]-CCACG-GGAGGAATAC-CAACC-CAGTG-3'-[6-carboxytetramethyl-rhodamine (TAMRA)]) (OIE, 2012).

\section{3 | RESULTS}

A total of 28,729 arthropods were caught from 14 pig farms with ASF outbreaks. The median number of arthropods collected per farm was 1,047 (minimum, 341; maximum, 11,359). The time interval between the ASF outbreak and arthropod collection varied from 1 to 44 days. In two farms, collection was performed during the onfarm outbreak investigation.

TABLE 1 Number of arthropods collected on pig farms with ASF outbreak, by order and region

\begin{tabular}{|c|c|c|c|c|c|c|c|c|c|c|}
\hline \multirow{2}{*}{$\begin{array}{l}\text { Region } \\
\text { Order }\end{array}$} & \multicolumn{2}{|l|}{$\begin{array}{l}\text { Paju } \\
\text { (5 farms) }\end{array}$} & \multicolumn{2}{|c|}{$\begin{array}{l}\text { Yeoncheon } \\
\text { ( } 2 \text { farms) }\end{array}$} & \multicolumn{2}{|l|}{$\begin{array}{l}\text { Gimpo } \\
\text { (2 farms) }\end{array}$} & \multicolumn{2}{|l|}{$\begin{array}{l}\text { Ganghwa } \\
\text { (5 farms) }\end{array}$} & \multicolumn{2}{|c|}{$\begin{array}{l}\text { Total } \\
\text { (14 farms) }\end{array}$} \\
\hline & Number & (\%) & Number & (\%) & Number & (\%) & Number & (\%) & Number & (\%) \\
\hline Diptera & 3,300 & (98.6) & 13,642 & (99.8) & 1,707 & (98.2) & 9,859 & (98.9) & 28,508 & (99.2) \\
\hline Blattaria & 0 & - & 20 & (0.1) & 3 & $(0.2)$ & 1 & $(<0.1)$ & 24 & $(<0.1)$ \\
\hline Ixodidae & 0 & - & 0 & - & 0 & - & 11 & (0.1) & 11 & $(<0.1)$ \\
\hline Coleoptera & 1 & $(<0.1)$ & 0 & - & 0 & - & 0 & - & 1 & $(<0.1)$ \\
\hline Unclassified & 45 & (1.3) & 10 & (0.1) & 28 & (1.6) & 97 & (1.0) & 180 & (0.6) \\
\hline Total & 3,346 & (100) & 13,672 & (100) & 1,738 & (100) & 9,973 & 100 & 28,729 & (100) \\
\hline
\end{tabular}

TABLE 2 Number of insects belonging to the order Diptera, by family and region, collected on pig farms with ASF outbreak

\begin{tabular}{|c|c|c|c|c|c|c|c|c|c|c|}
\hline \multirow{2}{*}{$\begin{array}{l}\text { Region } \\
\text { Family }\end{array}$} & \multicolumn{2}{|l|}{$\begin{array}{l}\text { Paju } \\
\text { (5 farms) }\end{array}$} & \multicolumn{2}{|c|}{$\begin{array}{l}\text { Yeoncheon } \\
\text { ( } 2 \text { farms) }\end{array}$} & \multicolumn{2}{|l|}{$\begin{array}{l}\text { Gimpo } \\
\text { (2 farms) }\end{array}$} & \multicolumn{2}{|l|}{$\begin{array}{l}\text { Ganghwa } \\
\text { (5 farms) }\end{array}$} & \multicolumn{2}{|c|}{$\begin{array}{l}\text { Total } \\
\text { (14 farms) }\end{array}$} \\
\hline & Number & (\%) & Number & (\%) & Number & (\%) & Number & (\%) & Number & (\%) \\
\hline Muscidae & 1,770 & $(53.6)$ & 11,464 & (84.0) & 1,181 & $(69.2)$ & 7,144 & $(72.5)$ & 21,559 & (75.6) \\
\hline Calliphoridae & 1,059 & (32.1) & 2,030 & (14.9) & 347 & (20.3) & 1,849 & 18.8 & 5,285 & (18.5) \\
\hline Culicidae & 297 & (9.0) & 51 & $(0.4)$ & 114 & (6.7) & 412 & 4.2 & 874 & (3.1) \\
\hline Tipulidae & 0 & - & 0 & - & 43 & (2.5) & 157 & 1.6 & 200 & $(0.7)$ \\
\hline Scathophagidae & 16 & $(0.5)$ & 15 & (0.1) & 1 & (0.1) & 112 & 1.1 & 144 & $(0.5)$ \\
\hline Sarcophagidae & 25 & $(0.8)$ & 33 & $(0.2)$ & 10 & $(0.6)$ & 40 & 0.4 & 108 & (0.4) \\
\hline Chironomidae & 0 & - & 0 & - & 10 & $(0.6)$ & 0 & - & 10 & $(<0.1)$ \\
\hline
\end{tabular}


The most common (99.2\%) arthropod collected belonged to the order Diptera (28,508 individuals from 10 families were collected; Table 1). In the order Diptera, the majority belonged to Muscidae ( $n=21,559,75.6 \%)$ and Calliphoridae ( $n=5,285,18.5 \%$; Table 2). Most Muscidae flies were Musca domestica (houseflies, $n=21,553$ ); however, Stomoxy calcitrans (stable flies, $n=6$ ) were also collected from three farms (Table S1). In addition, small numbers of Blattaria $(n=24)$, Ixodidae $(n=11)$, Lepidoptera $(n=5)$ and Coleoptera $(n=1)$ were collected from some regions. Furthermore, 108 (0.6\%) specimens could not be classified (Table 1). All the 28,729 collected arthropods were negative for ASFV genomic DNA.

\section{4 | DISCUSSION}

ASFV is known to persist for extended periods in environmental conditions and wild boar carcasses with the colonization of necrophagous pests, mainly belonging to the orders Diptera and Coleoptera (Bonnet et al., 2020). Experimental studies have reported the possibility of the mechanical transmission of ASFV by stable flies (Stomoxys calcitrans) and blowflies (Calliphoridae) (Forth et al., 2018; Mellor et al., 1987). Stable flies that fed on ASFV-spiked blood had detectable ASFV levels at 3 and $12 \mathrm{hr}$ after feeding. Moreover, the pigs upon which these flies fed became infected with ASFV (Olesen, Hansen, et al., 2018; Olesen, Lohse, et al., 2018). Alternatively, ASFV was detected only on the surface of the Calliphoridae larvae (Lucilia sericata and Calliphora vicina), without replication evidence (Forth et al., 2018).

In Poland, ASFV DNA was detected in stable flies collected from a pig farm with an ASF outbreak (Mazur-Panasiuk et al., 2019). In Estonia, ASFV DNA was detected in two of the flies (one of nine M. domestica and one of four Drosophila spp.) and pooling of two mosquitoes, which were randomly caught in the affected unit during an epidemiological investigation of a farm with an ASF outbreak. However, high Ct (threshold cycles) values indicated that only very small quantities of the virus were present (Herm et al., 2020). In Korea, there might be little possibility of missing positive specimen due to pooling. Same kind of arthropods collected at the same time at the same place were pooled into one sample. Therefore, the arthropod specimens included in the pooled sample had a very homogeneous potential for exposure to ASFV. Moreover, due to the prompt action on the farms with ASF outbreak (Kim et al., 2021), arthropod specimens were collected after the completion of slaughtering, except for two farms from which arthropods were collected during the investigation. Furthermore, ASFV was not detected in any farm. Moreover, 3,335 specimens were collected from the environment of these 14 farms with ASF outbreak. ASFV genomic DNA was detected from one tube, a pool of swabs of faeces and feed on an individual cage at a backyard farm (data unpublished). This reflected that the environment of the farms with ASF has not been extensively contaminated (Cho et al., 2021). Thus, a little possibility for arthropods to be exposed to ASFV was noted.

\section{5 | CONCLUSIONS}

In this study, a total of 28,729 arthropods were collected from farms with ASF outbreaks. No trace of ASFV genomic DNA was detected by laboratory analysis. This result may reflect the effects of immediate control measures following the detection of farms with ASF outbreaks in the early phase of infection in Korea (Cho et al., 2021; Kim et al., 2021; Yoon et al., 2020). The possibility of ASFV detection in arthropods collected from the farms with ASF outbreak cannot be ruled out if these were delayed.

\section{ACKNOWLEDGEMENT}

This study was supported by the Animal and Plant Quarantine Agency of the Republic of Korea [R\&D Project No.: I-1543068-2021-22-01].

\section{CONFLICT OF INTEREST}

None.

\section{ETHICS STATEMENT}

The authors confirm that the ethical policies of the journal, as noted on the journal's author guidelines page, have been adhered to. No ethical approval was required as this article is based on field work not involving any experiment.

\section{AUTHORS CONTRIBUTION}

Hachung Yoon*, DVM, PhD, Veterinary Epidemiology Division, Manuscript writing, Data analysis. Seong-Keun Hong, MS, Veterinary Epidemiology Division, Data analysis. Ilseob Lee, DVM, PhD, Veterinary Epidemiology Division, Data analysis. Deuksoo Choi, PhD, Plant Quarantine Technology Canter, Manuscript writing and review. Jongho Lee, PhD, Plant Pest Control Division, Manuscript review. Eunesub Lee, DVM, PhD, Veterinary Epidemiology Division, Planning and Overall operation. Sung-Hwan Wee, DVM, PhD, Department of Animal Disease Control and Quarantine, Conceptualization.

\section{PEER REVIEW}

The peer review history for this article is available at https://publo ns.com/publon/10.1002/vms3.545.

\section{ORCID}

Hachung Yoon (iD https://orcid.org/0000-0002-7434-9462

\section{REFERENCES}

Bonnet, S. I., Bouhsira, E., De Regge, N., Fite, J., Etore, F., Garigliany, M. M., Jori, F., Lempereur, L., Le Potier, M. F., Quillery, E., Saegerman, C., Vergne, T., \& Vial, L. (2020). Putative role of arthropod vectors in African swine fever virus transmission in relation to their bioecological properties. Viruses, 12. https://doi.org/10.3390/v1207 0778

Cho, K. H., Kim, H. J., Kim, D. Y., Yoo, D., Nah, J. J., Kim, Y. J., \& Kang, H. E. (2021). Surveillance of ASF-infected pig farms from September to October 2019 in South Korea. Journal of Veterinary Science, 22, e26. https://doi.org/10.4142/jvs.2021.22.e26

Forth, J. H., Amendt, J., Blome, S., Depner, K., \& Kampen, H. (2018). Evaluation of blowfly larvae (Diptera: Calliphoridae) as 
possible reservoirs and mechanical vectors of African swine fever virus. Transboundary and Emerging Diseases, 65, e210-e213. https:// doi.org/10.1111/tbed.12688

Herm, R., Tummeleht, L., Jurison, M., Vilem, A., \& Viltrop, A. (2020). Trace amounts of African swine fever virus DNA detected in insects collected from an infected pig farm in Estonia. Veterinary Medicine and Science, 6, 100-104. https://doi.org/10.1002/vms3.200

Jo, Y. S., \& Gortazar, C. (2020). African swine fever in wild boar, South Korea, 2019. Transboundary and Emerging Diseases. https://doi. org/10.1111/tbed.13532

Kim, H. J., Cho, K. H., Lee, S. K., Kim, D. Y., Nah, J. J., Kim, H. J., Kim, H. J., Hwang, J. Y., Sohn, H. J., Choi, J. G., Kang, H. E., \& Kim, Y. J. (2020). Outbreak of African swine fever in South Korea, 2019. Transboundary and Emerging Diseases, 67, 473-475. https://doi.org/10.1111/ tbed.13483

Kim, Y. J., Park, B., \& Kang, H. E. (2021). Control measures to African swine fever outbreak: Active response in South Korea, preparation for the future, and cooperation. Journal of Veterinary Science, 22, e13. https://doi.org/10.4142/jvs.2021.22.e13

Lee, K. L., Choi, Y., Yoo, J., Hwang, J., Jeong, H. G., Jheong, W. H., Kim, S. H. (2021). Identification of African swine fever virus genomic DNAs in wild boar habitats within outbreak regions in South Korea. Journal of Veterinary Science, 22, e28. https://doi.org/10.4142/jvs.2021.22.e28

Mazur-Panasiuk, N., Zmudzki, J., \& Wozniakowski, G. (2019). African swine fever virus - persistence in different environmental conditions and the possibility of its indirect transmission. Journal of Veterinary Research, 63, 303-310. https://doi.org/10.2478/jvetres-2019-0058

Mellor, P. S., Kitching, R. P., \& Wilkinson, P. J. (1987). Mechanical transmission of capripox virus and African swine fever virus by Stomoxys calcitrans. Research in Veterinary Science, 43, 109-112. https://doi. org/10.1016/S0034-5288(18)30753-7

OIE (2012). Manual of diagnostic tests and vaccines for terrestrial animals (mammals, birds and bees).

Olesen, A. S., Hansen, M. F., Rasmussen, T. B., Belsham, G. J., Bodker, R., $\&$ Botner, A. (2018). Survival and localization of African swine fever virus in stable flies (Stomoxys calcitrans) after feeding on viremic blood using a membrane feeder. Veterinary Microbiology, 222, 25-29. https://doi.org/10.1016/j.vetmic.2018.06.010

Olesen, A. S., Lohse, L., Hansen, M. F., Boklund, A., Halasa, T., Belsham, G. J., Rasmussen, T. B., Botner, A., \& Bodker, R. (2018). Infection of pigs with African swine fever virus via ingestion of stable flies (Stomoxys calcitrans). Transboundary and Emerging Diseases, 65, 1152-1157.

Petrasiunas, A., Bernotiene, R., \& Turcinaviciene, J. (2018). Catches of blood feeding flies with NZI traps in African swine fever affected areas of Lithuania. Bull Lithuanian Entomol Soc, 2, 112-118.

Vergne, T., Guinat, C., \& Pfeiffer, D. U. (2020). Undetected Circulation of African Swine Fever in Wild Boar, Asia. Emerging Infectious Diseases, 26, 2480-2482. https://doi.org/10.3201/eid2610.200608

Yoo, D., Kim, H., Lee, J. Y., \& Yoo, H. S. (2020). African swine fever: Etiology, epidemiological status in Korea, and perspective on control. Journal of Veterinary Science, 21, e38. https://doi.org/10.4142/ jvs.2020.21.e38

Yoon, H., Hong, S. K., Lee, I., Yoo, D. S., Jung, C. S., Lee, E., \& Wee, S. H. (2020). Clinical symptoms of African swine fever in domestic pig farms in the Republic of Korea, 2019. Transboundary and Emerging Diseases. https://doi.org/10.1111/tbed.13552

\section{SUPPORTING INFORMATION}

Additional supporting information may be found online in the Supporting Information section.

How to cite this article: Yoon $\mathrm{H}$, Hong S-K, Lee I, et al. Arthropods as potential vectors of African swine fever virus outbreaks in pig farms in the Republic of Korea. Vet Med Sci. 2021;7:1841-1844. https://doi.org/10.1002/vms3.545 